\title{
Lassalinho - Um agente pedagógico animado em um ambiente multiagente para Educação a Distância
}

\author{
Romualdo Santos Gomes* \\ Débora Nice Ferrari Barbosa ** \\ Cláudio Fernando Resin Geyer***
}

\begin{abstract}
Resumo. As ferramentas de comunicação síncronas estão dentre as várias possibilidades de comunicação em ambientes de educação a distância. Nesta categoria inclui-se a ferramenta jXChat e BonoBot. É esperado que os alunos sintam-se auto-motivados e que evitem a observação passiva neste tipo de ferramenta. Este trabalho propõe um agente pedagógico animado integrado a um chat e com comportamento de um chatterbot, capaz de interagir com o tutor ou aluno, de forma a auxiliar no processo de ensino e aprendizagem no ambiente virtual SEMEAI.
\end{abstract}

Palavras-chave - agente pedagógico animado, comunicação assíncrona, ambientes virtuais

\begin{abstract}
The synchronic communication tools are important possibilities of communication in Virtual Education Systems. The tool called "jxchat" is inside in this category. The virtual environments involve the students using this kind of tools, because they have needed sharing knowledge and experiences for the learning process. This paper propos Lassalinho - a pedagogical agent with the features of "chatterbot" that may helps the teaching-learning process in SEMEAI.
\end{abstract}

Keywords - pedagogical agent, assynchronous communications, virtual education system

\section{Introdução}

A Internet se tornou uma ferramenta poderosa que chegou com a proposta de globalizar a informação com rapidez e agilidade. Se bem utilizada, a Internet permitirá cada vez mais que as pessoas, independentes de sua classe social, credo ou posição política

\footnotetext{
"Bacharel em Ciência da Computação

Curso de Ciência da Computação/UNILASALLE

Bolsista - romualdo.gomes@terra.com.br

** Mestre/Doutoranda PPGC/UFRGS

Curso de Ciência da Computação/UNILASALLE

Orientadora - nice@unilasalle.edu.br

${ }^{* * *}$ Doutor

PPGC/UFRGS

Co-Orientador - geyer@inf.ufrgs.br
}

V. $3 \mathrm{~N}^{\mathrm{o}}$ 1, Maio, 2005 
tenham acesso à informação e principalmente adquiram e compartilhem o conhecimento. $\mathrm{O}$ mercado de trabalho impõe a necessidade de competências múltiplas do indivíduo, no trabalho em equipe, na capacidade de aprender e re-aprender e no aprendizado contínuo e, principalmente, em adaptar-se a situações novas. Neste contexto, a educação a distância $(\mathrm{EaD})$ consiste em uma nova proposta de educação para atender as novas demandas educacionais desta globalização que deixa de ser fenômeno sócio-econômico, hoje, para ser um fenômeno de transformação espacial e temporal (Belloni, 1997).

Com a aplicação da informática na educação, o paradigma tempo-espaço pode ser rompido, e a EaD vislumbra um futuro cada vez mais promissor. As aulas presenciais continuarão tendo a sua importância, mas com a EAD cria-se uma nova alternativa para o ensino-aprendizagem. A partir desse novo contexto, qualquer pessoa com um computador conectado à Internet passa a ter acesso ao fluxo de informação, ao processo de aprendizagem e o que é mais importante, torna-se um auto-gestor na construção do conhecimento.

Um dos grandes desafios da EaD é como fazer a colaboração e a cooperação em ambientes para $\mathrm{EaD}$. A Inteligência Artificial (IA) é a área que mais tem contribuído com pesquisas e construção de ferramentas no apoio ao ensino-aprendizagem. A IA tenta modelar o desempenho humano para melhor explorar o conhecimento que pode-se obter do indivíduo (Rich, 1993).

Com a incorporação das técnicas de IA no projeto e implementação desses sistemas, tem-se os STI (Sistemas Tutores Inteligentes), que simulam o processo de compreensão do raciocínio humano (pensamento) dentro de um domínio específico para auxiliar nas estratégias de solução de um problema ou na tomada de decisão (Giraffa, 1999). Estes softwares podem ser desenvolvidos utilizando a tecnologia de agentes, e podem constituir uma motivação a mais para os usuários de computadores, uma vez que, de acordo com Riecken (1994), "a idéia básica de Agentes Inteligentes é a construção de software que auxilie todos os tipos de usuários", ou seja, entende-se que a utilização de agentes inteligentes implica na ampliação da interatividade proporcionada pela máquina.. Com a incorporação desses agentes inteligentes a um ambiente ensinoaprendizagem, tem-se os chamados agentes pedagógicos. Os agentes pedagógicos são assim referidos por apresentarem um modelo definido por estratégias pedagógicas (Vassileva, 1997), (Geyer, 2001).

Com o emprego da tecnologia de agentes, é possível a construção e implementação de ambientes personalizados e mais colaborativos no auxílio de aprendizagem de alunos e professores. Como exemplo, pode-se mencionar o SEMEAI - Sistema Multiagente de Ensino Aprendizagem na Internet - que tem como objetivo selecionar estratégias de ensino, levando em consideração diferentes perfis de aluno (Geyer, 2001). Sendo assim, a ferramenta jXchat (Martins, 2003) foi desenvolvida como sendo um chat para ambientes de ensino virtual, que identifica e motiva o aluno a participar e, principalmente, fornecer ao professor um feedback. Para apoiar o uso do SEMEAI, também foi desenvolvida a ferramenta BonoBot (Sganderlla, 2003). Essa ferramenta é um Chatterbot, que visa a auxiliar o aluno em sua interação com o sistema, incentivando o aluno nos estudos e pesquisas e colaborando na construção do conhecimento, desempenhando assim um papel de monitor de estudo.

Diante desse contexto, este trabalho propõe integrar as qualidades, funcionalidades e conceitos das ferramentas $\mathrm{jXchat}$ e BonoBot, como forma de agregar conhecimentos específicos a um mesmo ambiente. A ênfase está na utilização de um agente 
pedagógico animado denominado Lassalinho que tem o objetivo de auxiliar no processo de ensino e aprendizagem do aluno através de uma ferramenta de chat integrada a um chatterbot. Assim, usando o $\mathrm{jXchat,}$ o aluno interage de forma virtual e, de forma integrada e implícita, pode "conversar" com um chatterbot para tirar dúvidas relacionadas ao tema em debate. Desta forma, o agente é capaz de auxiliar no processo de ensino e aprendizagem no ambiente virtual SEMEAI.

\section{Agente Pedagógico Animado Lassalinho}

Um dos objetivos principais do Lassalinho é aumentar a interatividade da ferramenta jXchat (Martins, 2003) com o usuário. Para tanto, em vez de motivar a participação do aluno através do envio de mensagens motivadoras a este, este trabalho propõe uma interatividade do agente pedagógico animado que terá um comportamento de um chatterbot com o aluno. O resultado dessa troca de interação irá gerar um feedback a ser enviado ao professor, sendo este capaz de definir as dificuldades que o estudante tenha encontrado no aprendizado de determinado assunto durante a interação no chat.

Outro papel a ser desempenhado pelo agente pedagógico animado é no auxílio ao processo de construção do conhecimento do aluno, devido ao agente poder acessar uma base de conhecimento do assunto a ser abordado no ambiente virtual. Com isso, o Lassalinho atuará também como um tutor, tendo o aluno portanto mais um suporte tecnológico para apoiar o ensino e aprendizagem no ambiente virtual SEMEAI. O ponto importante do trabalho proposto é possibilitar interações mais naturais e mais próximas entre os alunos e o ambiente de chat, em que a iniciativa de interação é normalmente compartilhada entre o ambiente virtual e o tutor.

\subsection{Modelo Funcional do Sistema}

O Lassalinho pode ser considerado um personagem "vivo" que coabita no ambiente virtual, no contexto da ferramenta $\mathrm{jXchat}$, interagindo com o aluno e gerando feedback ao professor. Essa interação explora a comunicação de forma a criar um ambiente de feedback interativo e dinâmico. Por esta razão, o agente tem um comportamento de um chatterbot, isto é, simula uma conversa através da troca de mensagens de texto, semelhante aos bate-papos virtuais. A figura 01 apresenta a figura do personagem. Por ter sido desenvolvido dentro do Centro Universitário La Salle, o mesmo é baseado no personagem representativo de La Salle para séries do ensino fundamental das Escolas Lassalistas.

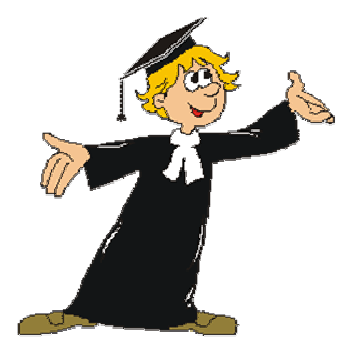

\section{Figura 01. Agente Pedagógico Animado Lassalinho}

Para realizar o tratamento das mensagens, optou-se pela técnica utilizada pela ferramenta BonoBot, ou seja a utilização de arquivos de definição em XML (eXtesible Markup Language) padrão para a representação e troca de dados na Internet. Com essa 
técnica é possível desenvolver uma própria linguagem de marcação. Em suma, o documento XML conterá a formatação das mensagens de entrada e saída que são respectivamente as mensagens recebidas do aluno e retorno para o mesmo.

O modelo cliente-servidor da Web é a base para o funcionamento do Lasallinho que executa no servidor, deixando a parte do cliente leve, não causando demora no processamento das páginas e permitindo assim o contínuo desenvolvimento dos seus sistemas. Permite também a independência do sistema operacional, bastando ao usuário ter acesso à Internet e ter um navegador instalado em sua máquina.

A figura 02 abaixo apresenta o modelo funcional do Lassalinho, mostrando a integração das funcionalidades das ferramentas já abordadas.

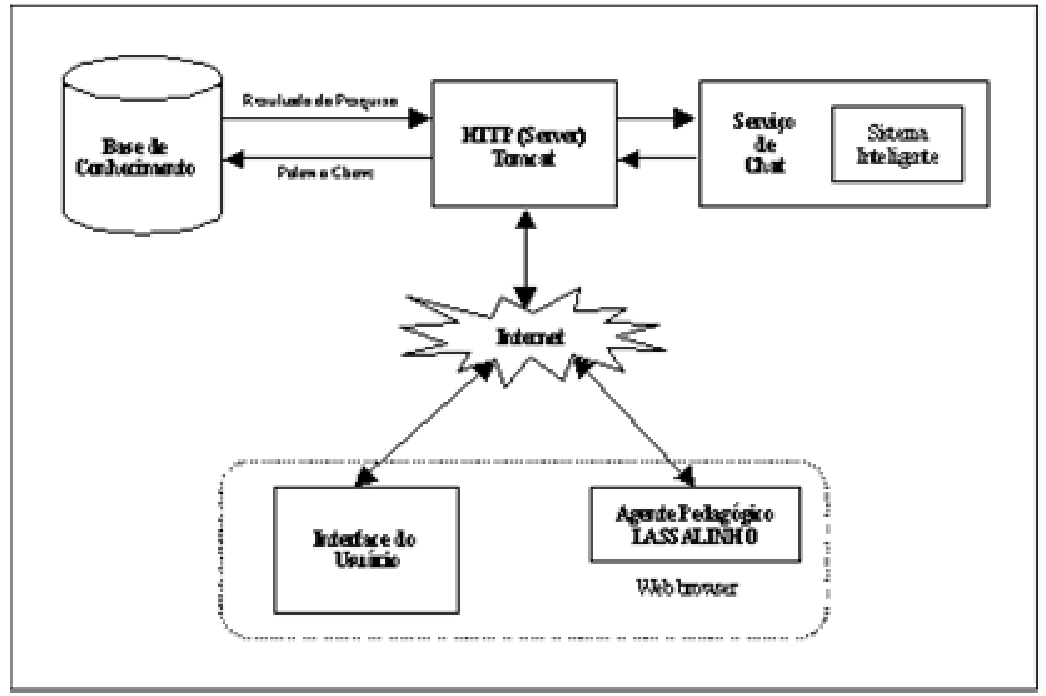

Figura 02. Modelo Funcional do Sistema

\subsection{Arquitetura do Sistema}

$\mathrm{Na}$ concepção Piagetiana, para que um indivíduo possa construir determinados conceitos se faz necessário que haja interação com os objetos do ambiente, propiciando o desenvolvimento de esquemas mentais que favoreça ou que possibilite o aprendizado (Belloni, 1997). Se houver motivação interna a aprendizagem passa a ser uma construção do conhecimento e, fundamentalmente, torna a aprendizagem mais significativa.A partir dessa concepção o agente Lassalinho procura, ao conceber uma interação com $\mathrm{o}$ aluno, auxiliar a aprendizagem e principalmente assegurar $\mathrm{o}$ encaminhamento da aprendizagem personalizada.

O usuário através de uma interface interage com o ambiente de chat juntamente com os demais. Caso algum dado referente ao aluno durante a interação no chat seja objeto de dúvida ou de necessidade de maiores embasamentos junto ao material, o aluno poderá interagir com o agente pedagógico animado e sua base de conhecimento. Ao interagir com o agente, o mesmo processa a entrada e compara com o conhecimento armazenado em sua base (comportando-se como um chatterbot). Se o conhecimento existir, o agente repassa ao usuário através da interface de diálogo e a conversa pode continuar normalmente. Caso o conhecimento não exista na base, o agente sugere ao aluno formas de aquisição deste conhecimento.

O agente pedagógico, além de proporcionar a visão de um "personagem vivo", proporciona a possibilidade de conversa "particular" sobre um determinado tema. Este 
aspecto, durante uma sessão de chat, pode fazer com que alunos que estão em dificuldades de compreender alguns aspectos tratados, possam ser apoiados pelo agente, sendo portanto incentivados a participar da conversa virtual. Além disto, o agente constantemente motiva os alunos menos participativos com mensagens animadoras, trazendo os mesmos para o processo de interação. A figura 03 apresenta de forma mais detalhada a arquitetura do sistema.

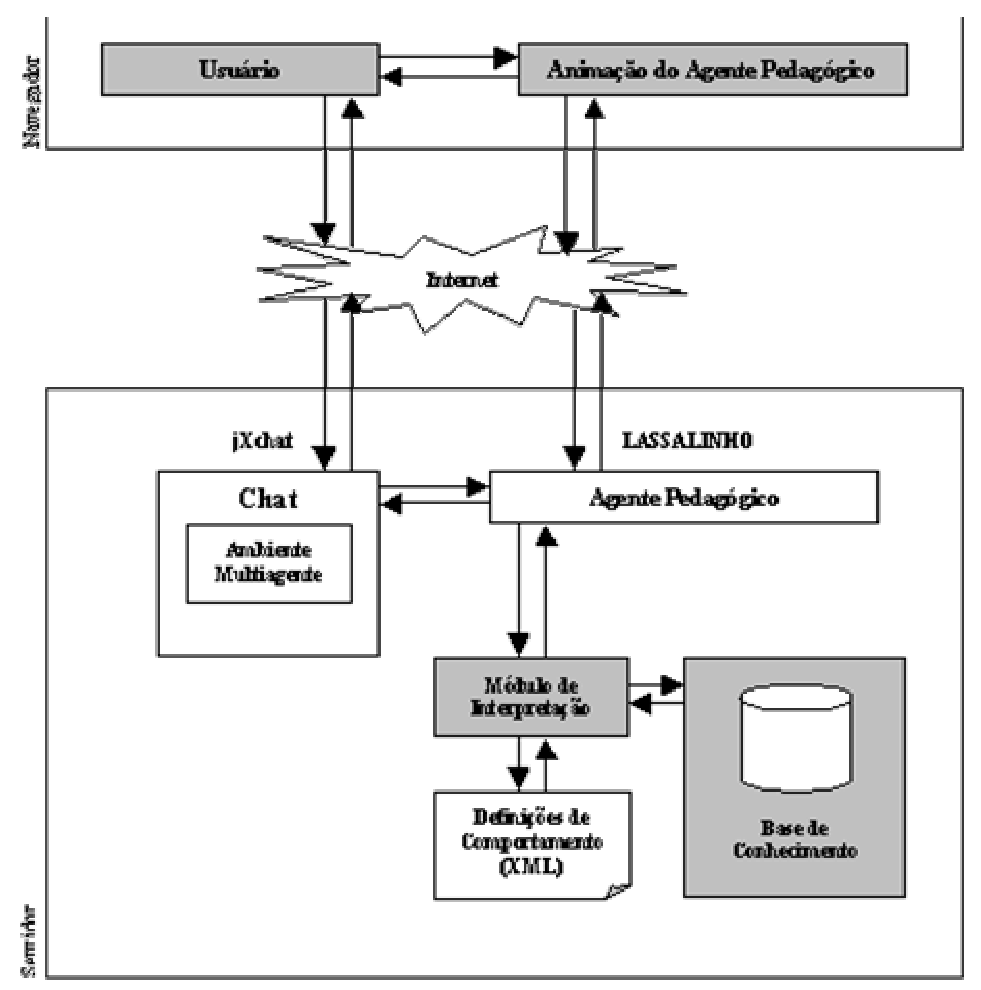

Figura 03. Arquitetura do Sistema

Os elementos em destaque mostram os módulos do sistema que foram alterados para suportar a integração e para agregar as características do Lassalinho. Destaca-se, dentre outras, as principais alterações:

- Inserção de um módulo cliente para suportar as animações do agente Lassalinho;

- Alteração no servidor de chat de forma que o aluno, ao interagir com o agente, tenha o seu nome presente na tela de interação;

- Nova formatação do módulo de interpretação com a adição de novas categorias, com o objetivo de melhorar a dinâmica de interação entre o agente e o aluno e inserção da "personalidade" do agente Lassalinho;

- Inserção de novas funcionalidades para que o professor possa atualizar a base de conhecimento levando em consideração o feedback apresentado pelo agente;

- Alteração do feedback dado pelo sistema ao professor, incorporando a possibilidade do mesmo acessar a o que foi "conversado" com o Lassalinho durante a seção de chat. 


\section{Aspectos de Implementação e Validação}

Utilizando tecnologias de livre acesso, a linguagem Java foi utilizada para desenvolvimento e o banco de dados PostgreSQL Database Server 8.0 para o armazenamento da base de conhecimento. Para suporte a web foram utilizados o JSP e o servidor Apache Jakarta Tomcat 4.1.2. Para a construção do personagem representativo animado do agente foi utilizada a ferramenta Gimp e software freeware da Microsoft, o Microsoft Gif Animator. A figura 04 apresenta uma das interfaces do agente dentro do contexto do chat e com o comportamento de um chatterbot (figura sobreposta).

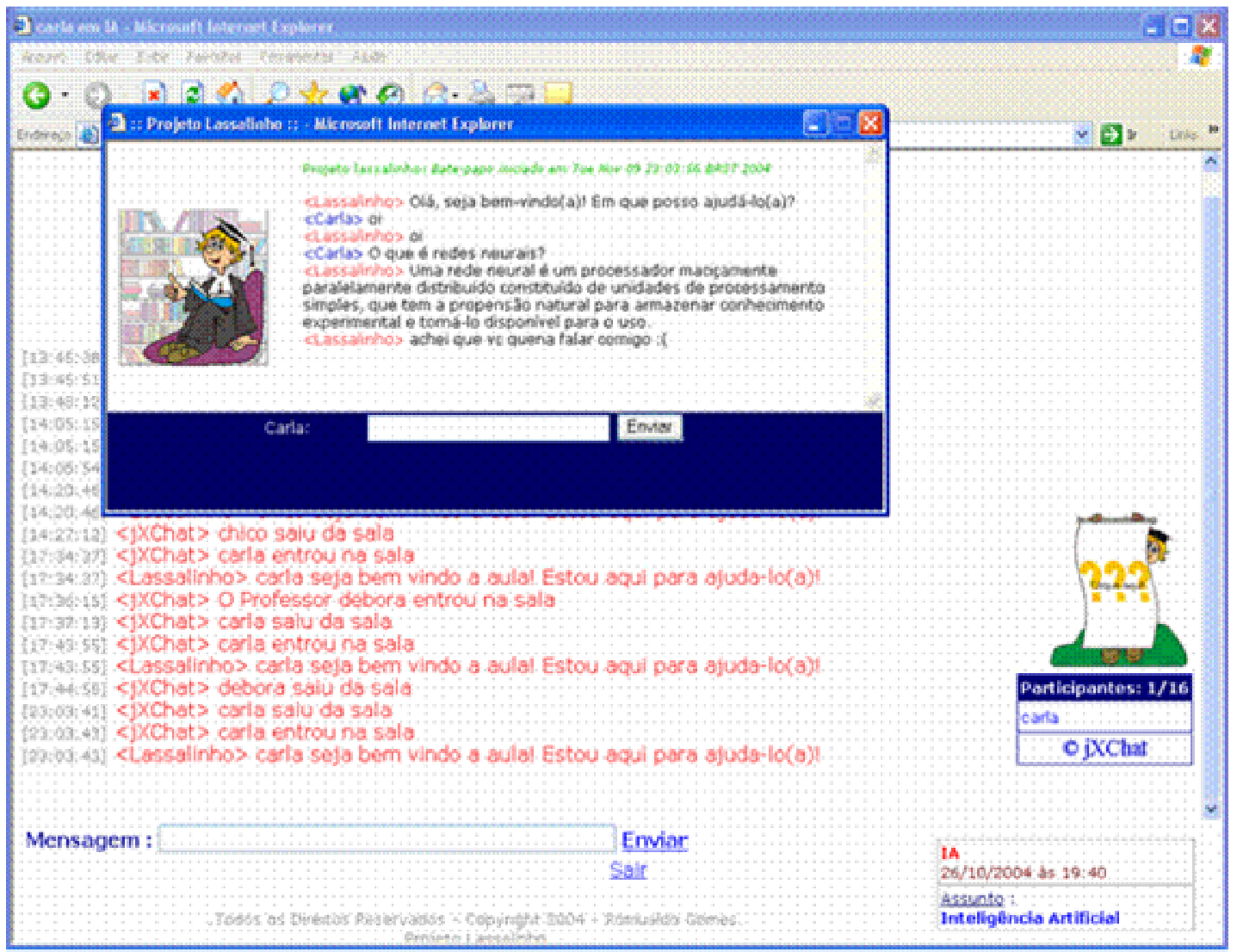

Figura 04. Interfaces do sistema

A proposta foi valida junto aos alunos do curso da Ciência da Computação, mais especificamente da disciplina de Inteligência Artificial, do UNILASALLE. Os alunos efetivamente fizeram a interação com o sistema. Para não comprometer a validação foi evitado o auxílio ao usuário. Após finalização dos testes, dentro de um período prédeterminado, os alunos foram convidados a comentar, sugerir e responder um questionário. As coletas de dados foram feitas através da observação direta ao usuário utilizando técnicas de anotação de dados do tipo checklist e da análise dos questionários que cada aluno preencheu após o término dos testes.

A análise dos resultados comprovou que é válida a inserção de um agente pedagógico animado em um ambiente interativo como o $\mathrm{jXchat}$. O ponto alto do trabalho foram as animações do personagem Lassalinho. Segundo Giraffa (1999), a utilização de agentes animados em sistemas de ensino e aprendizagem proporciona um ambiente mais agradável, divertido e estimulante, permitindo assim, um ganho de qualidade sob o 
ponto de vista pedagógico. Uma observação, segundo avaliação dos alunos, foi referente a desenvoltura do agente Lassalinho em sua interação com o usuário como um chatterbot. Sganderla (2003) já mencionava essa dificuldade em seu trabalho e mesmo com o acréscimo de categorias de comportamento ao agente pedagógico para melhorar a sua desenvoltura, a interação com o aluno ficou ainda muito limitada. $\mathrm{O}$ trabalho de Van Lare (2004) procura amenizar estas questões.

\section{Conclusão}

Martins (2003) em um estudo mais aprofundado defende o uso de salas de chat em ambiente de $\mathrm{EaD}$, mas ressalta que é preciso uma técnica pedagógica correta, elementos internos motivadores, recursos didáticos capazes de fazer despertar nos alunos e nos professores a aceitação desse novo paradigma e além da capacidade técnica inteligente de estabelecer uma relação de incentivo à participação do aluno e auxílio ao professor.

O presente trabalho propôs validar o uso de agente pedagógico animado em ferramenta síncrona de chat inserida no ambiente virtual SEMEAI, visando criar condições para que os alunos se sintam menos isolados ao participarem de atividades que usam este tipo de comunicação. Nesse contexto, com a validação do trabalho, a utilização do agente pedagógico teve a finalidade de aprimorar a interação neste ambiente e de fornecer informações mais personalizada de cada aluno. $\mathrm{O}$ agente, além de proporcionar a visão de um "personagem vivo", proporciona a possibilidade de conversa "particular" sobre um determinado tema. Além disto, constantemente motiva os alunos menos participativos com mensagens animadoras, trazendo os mesmos para o processo de interação. Não se tem conhecimento na literatura de trabalhos que integram as potencialidades das ferramentas de chat e chatterbot.

Acredita-se que a realização deste trabalho contribuiu para a busca de alternativas que apóiem os processos de ensino e aprendizagem através de ambientes virtuais, aproximando a teoria da prática, e tornando o ambiente mais interativo, participativo e interessante. $\mathrm{O}$ estudo feito ao longo do trabalho permitiu a proposição de melhorias. A perspectiva de realização de novos experimentos, agregando o proposto no trabalho de Van Lare (2004) e a validação com turmas do ensino fundamental, é a principal motivação para a continuidade deste trabalho.

\section{Agradecimentos}

Os autores agradecem ao UNILASALLE pelo apoio financeiro no desenvolvimento deste trabalho e do ambiente SEMEAI. 


\section{Referencias Bibliográficas}

BELLONI, M. L. (1997). Educação a distância. Brasília: XVI Jornada de Atualização em Informática.

RICH, E; KNIGHT, K. (1993). Inteligência Artificial. São Paulo: Makron Books. $722 \mathrm{p}$.

RIECKEN, D.; MINSKY, M. A Conversation with Marvin Minsky About Agents. In: COMMUNICATIONS OF THE ACM 1994, Vol. 37, nr. 7, pp. 22-29.

VASSILEVA, J. Goal-Based Pedagogical Agents. WORKSHOP PEDAGOGICAL AGENT, Proceeding.., Kobe, Japan, 1997.

GEYER,C. F; FERRARI, D. N, et al. SEMEAI - Sistema Multiagente de Ensino Aprendizagem na Internet. In: XII SIMPÓSIO BRASILEIRO DE INFORMÁTICA NA EDUCAÇÃO (SBIE), Vitória, 2001.

MARTINS, J. F.; FERRARI, D.N. Sistema de Comunicação Eletrônica Inteligente em um Ambiente Multiagente para Ensino a Distância. In: XII SIMPÓSIO BRASILEIRO DE INFORMÁTICA NA EDUCAÇÃO (SBIE), Rio de Janeiro, (2003).

SGANDERLLA, R. B.; FERRARI, D.N. BonoBOT: Um Chatterbot para Interação com Usuários em um Sistema Tutor Inteligente. In: XII XII SIMPÓSIO BRASILEIRO DE INFORMÁTICA NA EDUCAÇÃO (SBIE), Rio de Janeiro, 2003.

GIRAFFA,L. M., VICARI, R.. The Use of Agents Techniques on Intelligent Tutoring Systems. In: International Conference of the Chilean Computer Science Society (1999)

VAN LARE, N. J.; Uma Proposta de Processamento de Linguagem Natural para o BonoBot. Canoas: Centro Universitário La Salle, 2004, 97p. Trabalho de Conclusão. 